VOL. 24 (1981), 373-379.

\title{
EMBEDDING GROUP AMALGAMS
}

\section{Patrick Fitzpatrick and James Wiegold}

\begin{abstract}
A class $\underline{\underline{X}}$ of groups is said to have the small embedding property if every amalgam of two groups from $\underline{\underline{X}}$ amalgamating a normal subgroup embeds into a group not generating the variety $\underline{\underline{0}}$ of all groups. Very few large classes have the small embedding property. For example, the minimal non-abelian varieties containing non-abelian finite groups do not; neither does any class of groups containing $\mathrm{F}_{2}\left(\underline{\underline{N}}_{2}\right)$; but the set of f'initely generated groups in a nilpotent variety not containing $\stackrel{\mathrm{N}}{2}_{2}$ does have the small embedding property.
\end{abstract}

An amalgam $A=\operatorname{am}(A, B ; H)$ of groups $A$ and $B$ amalgamating $H=A \cap B$ is said to be normal if $H$ is normal in $A$ and in $B$. We shall say that a class $X$ of groups has the small embedding property if every normal amalgam of groups $A$ and $B$ from $\underline{\underline{X}}$ can be embedded in $a$ group not generating the variety $\underline{\underline{O}}$ of all groups. Clearly, every class of finite groups has the small embedding property, but our first result shows how soon one meets trouble.

THEOREM 1. No class of groups containing the free group of rank 2 of $\underline{\underline{N}}_{2}$ has the small embedding property.

Proof. Here, and in what follows, notation is that of Hanna Neumann's book [2].

Let

Received 16 June 1981. 


$$
\begin{aligned}
& A=\langle a, b \mid[a, b, a]=[a, b, b]=1\rangle, \\
& B=\langle c, d \mid[c, d, c]=[c, d, d]=1\rangle,
\end{aligned}
$$

be free nilpotent of class 2 , and form the amalgam am $(A, B ; H)$ by identifying the subgroups $H=\langle a,[a, b]\rangle$ and $H \theta=\langle c,[c, d]\rangle$ according to the isomorphism $\theta$ defined by $a \theta=[c, d],[a, b] \theta=c$. Let $G$ be any group generated by this amalgam. Then the group of automorphisms induced by $G$ on $H$ contains the automorphisms $\phi, \psi$ defined by

$$
\begin{aligned}
a \phi & =a^{b}=a[a, b], \\
{[a, b] \phi } & =[a, b]^{b}=[a, b], \\
a \psi & =a^{d}=[c, d]^{d}=[c, d]=a, \\
{[a, b] \psi } & =[a, b]^{d}=c^{d}=c[c, d]=a[a, b] .
\end{aligned}
$$

Now $H$ is free abelian of rank 2 , and it follows that $G$ has as homomorphic image the subgroup of $\operatorname{Aut}(H)=\mathrm{SL}(2, Z)$ generated by the matrices $\left(\begin{array}{ll}1 & 1 \\ 0 & 1\end{array}\right)$ and $\left(\begin{array}{ll}1 & 0 \\ 1 & 1\end{array}\right)$, that is, $\mathrm{SL}(2, Z)$ itself. Since every countable free group can be embedded in $\mathrm{SL}(2, Z), G$ generates $\underline{\underline{0}}$, as claimed.

It was this result, proved in response to a query of Glass, that set us off on this search in the first place. It shows that varieties with the small embedding property are likely to be very restricted; indeed, we conjecture that no non-abelian variety has the small embedding property. We have been unable to confirm this conjecture, mainly because of the possible existence of non-abelian varieties with all finite groups abelian, the so-called "pseudo-abelian" varieties. Every other non-abelian variety must contain a variety of the form $\underline{\underline{B}}_{4} \wedge \underline{\underline{N}}_{2}, \stackrel{B}{p}_{p} \wedge \underline{\underline{N}}_{2}(p$ an odd prime), or $\stackrel{A}{A}{ }_{q}(p, q$ different primes). These varieties are minimal in the sense that all proper subvarieties are abelian, so that our second result lends substance to our conjecture.

THEOREM 2. $\underline{\underline{B}}_{4} \wedge \stackrel{\mathbb{N}}{=}_{2}, \quad \underline{B}_{p} \wedge \underline{\underline{N}}_{2}$ and $\stackrel{A}{=} \stackrel{A}{A}$ all fail to have the small embedding property. 
Proof. For $\underline{\underline{B}}_{4} \wedge \stackrel{\mathbb{N}}{=}_{2}$, we start with two copies of the $n$th direct power of $D_{8}$ :

$$
\begin{aligned}
A_{n}=\left\langle a_{1}, b_{1} \mid a_{1}^{4}=b_{1}^{2}=\left(a_{1} b_{1}\right)^{2}=1\right\rangle & \times \\
& \times\left\langle a_{n}, b_{n} \mid a_{n}^{4}=b_{n}^{2}=\left(a_{n} b_{n}\right)^{2}=1\right\rangle, \\
B_{n}=\left\langle c_{1}, d_{1} \mid c_{1}^{4}=d_{1}^{2}=\left(c_{1} d_{1}\right)^{2}=1\right\rangle & \times \ldots \\
& \times\left\langle c_{n}, d_{n} \mid c_{n}^{4}=d_{n}^{2}=\left(c_{n} d_{n}\right)^{2}=1\right\rangle,
\end{aligned}
$$

and form the normal amalgam $\operatorname{am}\left(A_{n}, B_{n} ; H_{n}\right)$ by identifying the subgroups $H_{n}=\left\langle a_{1}^{2}, b_{1}\right\rangle \times \ldots \times\left\langle a_{n}^{2}, b_{n}\right\rangle$ and $H_{n} \theta=\left\langle c_{1}^{2}, d_{1}\right\rangle \times \ldots \times\left\langle c_{n}^{2}, d_{n}\right\rangle$

according to the isomorphism $\theta$ defined by

$$
a_{1}^{2} \theta=d_{1}, b_{1} \theta=c_{2}^{2}, \ldots, a_{n}^{2} \theta=d_{n}, b_{n} \theta=c_{1}^{2} .
$$

Let $G$ be any group generated by the amalgam. Then the group of automorphisms of $H_{n}$ induced by $G$ may be regarded as a subgroup of $G L(2 n, 2)$. It is an easy exercise to show that this subgroup contains all transvections and so is all of $\mathrm{GL}(2 n, 2)$.

Now form the direct products

$$
A=\prod_{n=1}^{\infty} A_{n}, \quad B=\prod_{n=1}^{\infty} B_{n}, \quad H=\prod_{n=1}^{\infty} H_{n},
$$

and the obvious amalgam $\operatorname{am}(A, B ; H)$. It follows that every group generated by the amalgam has $\prod_{n=1}^{\infty} \operatorname{GL}(2 n, 2)$ as an image, and so it must generate $\underline{O}$ (for one of the many ways of proving this, see Jones [1]).

The proof for $\stackrel{\mathrm{B}}{=}_{p} \wedge \stackrel{\mathrm{N}}{=}_{2}, p$ an odd prime, follows the same pattern.

Set

$$
\begin{aligned}
A_{n}=\left\langle a_{1}, b_{1}\right| a_{1}^{p} & \left.=b_{1}^{p}=\left[a_{1}, b_{1}, a_{1}\right]=\left[a_{1}, b_{1}, b_{1}\right]=1\right\rangle \times \ldots \\
& \times\left\langle a_{n}, b_{n} \mid a_{n}^{p}=b_{n}^{p}=\left[a_{n}, b_{n}, a_{n}\right]=\left[a_{n}, b_{n}, b_{n}\right]=1\right\rangle,
\end{aligned}
$$




$$
\begin{aligned}
& B_{n}=\left\langle c_{1}, d_{1}\right| c_{1}^{p}\left.=d_{1}^{p}=\left[c_{1}, d_{1}, c_{1}\right]=\left[c_{1}, d_{1}, d_{1}\right]=1\right\rangle \times \ldots . \\
& \times\left\langle c_{n}, d_{n} \mid c_{n}^{p}=d_{n}^{p}=\left[c_{n}, d_{n}, c_{n}\right]=\left[c_{n}, d_{n}, d_{n}\right]=1\right\rangle, \\
& H_{n}=\left\langle a_{1},\left[a_{1}, b_{1}\right]\right) \times \ldots \times\left\langle a_{n},\left[a_{n}, b_{n}\right]\right\rangle, \\
& H_{n} \theta=\left\langle c_{1},\left[c_{1}, d_{1}\right]\right\rangle \times \ldots \times\left\langle c_{n},\left[c_{n}, d_{n}\right]\right\rangle, \\
& a_{1} \theta=\left[c_{1}, d_{1}\right],\left[a_{1}, b_{1}\right] \theta=c_{2}, \ldots, a_{n} \theta=\left[c_{n}, a_{n}\right],\left[a_{n}, b_{n}\right] \theta=c_{1} .
\end{aligned}
$$

In this case, if $G$ is a group generated by the amalgam, the group of automorphisms of $H_{n}$ induced by $G_{n}$ is a subgroup of $G L(2 n, p)$ containing all transvections, and therefore it contains $\operatorname{SL}(2 n, p)$. The conclusion of the argument goes as before.

Finally, with $\stackrel{A}{\Rightarrow} \mathrm{A}_{q}$, we use a somewhat different approach. For every $n \geq 1$, it is clear that the following subgroups of $\operatorname{Sym}(n q)$ are elementary abelian $q$-groups, that is, they are in $\stackrel{\mathrm{A}}{\Rightarrow} q$

$$
\begin{aligned}
& x_{n}=\langle(1,2, \ldots, q),(q+1, \ldots, 2 q), \ldots,(n q-q+1, \ldots, n q)\rangle, \\
& Y_{n}=\langle(n q, 1, \ldots, q-1),(q, \ldots, 2 q-1), \ldots,(n q-q, \ldots, n q-1)\rangle .
\end{aligned}
$$

A simple calculation shows that $\left\langle X_{n}, Y_{n}\right\rangle=\operatorname{Alt}(n q)$ if $q$ is odd, $\left\langle X_{n}, y_{n}\right\rangle=\operatorname{Sym}(n q)$ if $q=2$. Now let $A_{n}$ be a split extension of an elementary abelian $p$-group $H_{n}$ of rank $n q$,

$$
H_{n}=\left\langle h_{1}, \ldots, h_{n q}\right\rangle \text {, }
$$

by $x_{n}$, with $x_{n}$ permuting the suffices $1, \ldots, n q$ of the generators according to the permutation representation of $X_{n}$ as displayed. Do the same thing with $Y_{n}$, that is, form the analogous split extension $B_{n}$ of

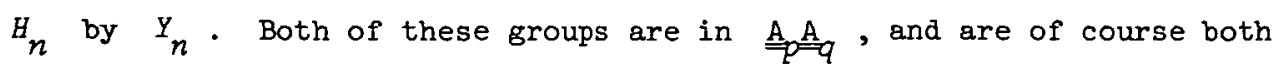
isomorphic with the permutational wreath product of a cyclic group of order $p$ by $X_{n}$. We form the obvious amalgam $\operatorname{am}\left(A_{n}, B_{n} ; H_{n}\right)$ and observe that if $G$ is a group generated by it, the group of automorphisms induced by $G$ on $H_{n}$ is $\mathrm{Alt}(n q)$ or $\operatorname{Sym}(n q)$. Proceed as before: set 


$$
A=\prod_{n=1}^{\infty} A_{n}, \quad B=\prod_{n=1}^{\infty} B_{n}, \quad H=\prod_{n=1}^{\infty} H_{n} .
$$

Every group generated by the amalgam $\operatorname{am}(A, B ; H)$ generates $\underline{\underline{0}}$ since it has arbitrarily large alternating or symmetric groups as images. This completes the proof of Theorem 2 .

After all these counterexamples it is not surprising that our only positive result is a weak one.

THEOREM 3. If $\underline{\underline{\mathrm{V}}}$ is a variety of nilpotent groups not containing $\underline{\underline{N}}_{2}$, then the class of finitely generated groups in $\underline{\underline{V}}$ has the small embedding property.

The proof is based on several lemmas. Henceforth $\mathrm{A}=\operatorname{am}(A, B ; H)$ is a normal amalgam of finitely generated groups from some nilpotent variety $\underline{V}$ which does not contain $\underline{\underline{N}}_{2}$, and $G$ is the generalised free product of A. Our aim is to show that a certain factor group of $G$ which does not generate $\underline{\underline{0}}$ embeds $A$.

LEMMA 1. The group of automorphisms induced by $G$ on $H$ is finite.

Proof. Under the given conditions each of $A$ and $B$ has finite derived group. The normal subgroups $[A, H]$ and $[B, H]$ of $H$ are therefore finite and hence so also is $[A, H][B, H]$. Each $g \in G$ acts as $h^{g}=h k$ where $h \in H$ and $k \in[A, H][B, H]$. Since $H$ is finitely generated there can only be finitely many distinct automorphisms of $H$ induced by elements of $G$, and this proves the lemma.

As a consequence there exists a positive integer $m$ such that $[A, B]^{m}$ centralizes $H$. By Theorem 2.1 of [3],

$$
[A, B] \cap H=[A, H][B, H] \text {, }
$$

so we can assert further that

$$
[A, B]^{m} \cap H \leq Z(H) \cap[A, H][B, H]
$$

and this latter group is finite. The next step is to show:

LEMMA 2. There exists a positive integer $n$ such that $[A, B]^{n} \cap H=1$. 
Proof. Let $S$ and $T$ be left transversals of $H$ in $A$ and $B$ respectively. With $m$ as above, each element $w$ of $[A, B]^{m}$ can be written as a product of commutators [sh, th'] with obvious notation. This can be expressed as $w=v u$ where $u$ lies in $[A, H][B, H]$ and $v$ is a product of integral powers of commutators of the form $[s, t]$ with in $S$ and $t$ in $T$. Since $w$ centralises $H$,

$$
w=(v u)\left(u u^{-1}\right)=u(v u) u^{-1}=u v \text {. }
$$

Thus if $e$ is the exponent of $[A, H][B, H]$, then $w^{e}=v^{e}$, and so each element of $[A, B]^{m e}$ can be expressed in the form

$$
\left[s_{1}, t_{1}\right]^{\varepsilon_{1}}\left[s_{2}, t_{2}\right]^{\varepsilon_{2}} \ldots\left[s_{k}, t_{k}\right]^{\varepsilon_{k}}
$$

with $s_{i}$ in $S, t_{i}$ in $T$ and the $\varepsilon_{i}$ integers. If this element lies in $H$, then using star to denote images in the factor group $G / H$,

$$
\left[s_{1}^{*}, t_{1}^{*}\right]^{\varepsilon_{1}} \ldots\left[s_{k}^{*}, t_{k}^{*}\right]^{\varepsilon_{k}}=1
$$

Clearly the terms in the original product can be arranged so that no consecutive elements $\left[s_{i}^{*}, t_{i}^{*}\right],\left[s_{i+1}^{*}, t_{i+1}^{*}\right]$ coincide. But $\left[A^{*}, B^{*}\right]$ is absolutely free on generators $\left[a^{*}, b^{*}\right]$ with $a \in S-H$ and $b \in T-H$. Hence each $\varepsilon_{i}$ is zero and $[A, B]^{m e} \cap H=1$, as required.

Next an adaptation of the argument of Theorem 2.2 of [3] gives the following result, which is independent of our assumptions on $A$ and $B$.

LEMMA 3. If $Y$ is a normal subgroup of $G$ which is contained in $[A, B]$ and intersects $H$ trivially, then $G / Y$ embeds the comalgam $A$.

Proof. Let $L=G / Y$. The image of $A$ in $L$ is isomorphic to $A /(A \cap Y)$, so let $a \in A \cap Y$. Again using star to denote images modulo $H, a^{*} \in A^{*} \cap Y^{*}$. But $G^{*}$ is the absolutely free product of $A^{*}$ and $B^{*}$ so $A^{*}$ intersects $\left[A^{*}, B^{*}\right]$ trivially. Thus $A^{*} \cap Y^{*}$ is trivial and $a^{*}=1$. This implies $a \in Y \cap H$, which by assumption is trivial. Hence $a=1$ and $A$ is isomorphically represented in $G$. Similarly for $B$. Now the intersection of $A Y / Y$ and $B Y / Y$ contains $H Y / Y$. Conversely, suppose $a Y=b Y$ with $a$ in $A, b$ in $B$ so that $a=b y$ for some $y$ 
in $y$. Then $a^{*}=b^{*} y^{*}$ so $a^{*}$ is in the normal closure of $B^{*}$ in $G^{*}$. Thus $a^{*}=1$ so $a \in H$ and the intersection of $A Y / Y$ and $B Y / Y$ is precisely $H Y / Y$. This completes the proof of the lemma.

We can now prove the theorem as follows. By Lemmas 2 and 3 the group $L=G /[A, B]^{n}$ embeds the amalgam. It remains to prove that $L$ does not generate $\underline{\underline{0}}$ and to this end we show that $L^{\prime}$ has finite exponent. Let $C$ and $D$ be the images in $L$ of $A$ and $B$ respectively and let $r$ denote the product of the (finite) exponents of $C^{\prime}$ and $D^{\prime}$. The factor group of $L^{\prime}=C^{\prime} D^{\prime}[C, D]$ by its subgroup $[C, D]$ is a central product of the images modulo $[C, D]$ of $C^{\prime}$ and $D^{\prime}$ and hence has exponent dividing $r$. Since $[C, D]$ itself has exponent dividing $n$, $L^{\prime}$ has finite exponent dividing $n r$. This completes the proof of the theorem.

\section{References}

[1] Gareth A. Jones, "Varieties and simple groups", J. Austral. Math. Soc. $17(1974), 163-173$.

[2] Hanna Neumann, Varieties of groups (Ergebnisse der Mathematik und ihrer Grenzgebiete, 37. Springer-Verlag, Berlin, Heidelberg, New York, 1967).

[3] James Wiegold, "Soluble embeddings of group amalgams", Publ. Math. Debrecen 12 (1965), 227-230.

Department of Mathematics, University College,

Cork,

Ireland;

Department of Pure Mathematics, University College,

PO Box 78,

Cardiff CFI $\mid X L$, wales. 